\title{
Acceleration Sensor-based First Resonance Vibration Suppression of a Double-clamped Piezoelectric Beam
}

\author{
Zhi-cheng Qiu \\ School of Mechanical and Automotive Engineering, South China University of Technology, Guangzhou 510641, \\ PR China \\ State Key Laboratory of Robotics, Shenyang Institute of Automation, Chinese Academy of Sciences, Shenyang \\ 110016, PR China
}

Fan-kong Meng

School of Mechanical and Automotive Engineering, South China University of Technology, Guangzhou 510641, PR China

\author{
Jian-da Han and Jin-guo Liu \\ State Key Laboratory of Robotics, Shenyang Institute of Automation, Chinese Academy of Sciences, Shenyang \\ 110016, PR China
}

\begin{abstract}
(Received 13 November 2013; accepted 12 March 2014)
This paper investigates resonance vibration suppression under persistent excitation near the first structural resonant frequency of a clamped-clamped (doubly-clamped) piezoelectric flexible beam. In this study, an acceleration sensor is used to measure the resonant vibration. Firstly, the finite element method (FEM) is utilized to derive the dynamics model of the system, and modal analysis is carried out. Secondly, an acceleration feedback-based proportional-integral control algorithm and variable structure control algorithms are designed, and a numerical simulation is performed. Finally, a doubly-clamped piezoelectric flexible beam experimental setup is constructed. Experiments are conducted on resonant vibration suppression using the designed control algorithms. The numerical simulation and experimental results demonstrate that the resonant vibration can be suppressed by using the designed control methods, and the improved variable structure control method shows better control performance in suppressing the resonant vibration.
\end{abstract}

\section{INTRODUCTION}

Flexible beam structures are characterized by light weight, low structural damping, and low modal frequencies, and they are one of the main structure types used in the engineering field and aerospace structures. ${ }^{1}$ Vibrations are easily caused when flexible beams are subjected to heavy loads or affected by a variety of unexpected external factors in the course of their work. Furthermore, these vibrations will last for a long time. If the vibrations are not suppressed effectively, the prolonged vibrations will reduce the working accuracy and working life of large and complex structures, such as space crafts and space robot manipulators. Moreover, if the structure is excited at its resonance frequencies, it will be seriously damaged. ${ }^{2}$ Therefore, active vibration control of flexible structures is an important concern.

During the past few decades, a considerable amount of study in the area of active vibration control of flexible structures has been conducted by many researchers. In their investigations, piezoelectric materials (such as PZT, PVDF) are widely used in active vibration control for flexible structures. They provide inexpensive, reliable, and non-intrusive means of actuating and sensing vibrations in flexible structures. ${ }^{4}$ Wang, et al., ${ }^{5}$ conducted a study on the vibration control of smart piezoelectric composite plates; they investigated the effect of the stretching- bending coupling of the piezoelectric sensor and actuator pairs on the system stability of smart composite plates. The classical negative velocity feedback control method was adopted for the active vibration control analysis of smart composite plates with bonded distributed piezoelectric sensors and actuators in their study. Sabatini, et al., ${ }^{6}$ studied active damping strategies and relevant devices that could be used to reduce the structural vibrations of a space manipulator with flexible links during its in orbit operations. They proposed an optimized adaptive vibration control via piezoelectric devices. Carlos, et al., ${ }^{7}$ conducted an experimental study into the control of a cantilever beam, which had a PZT patch bonded to it as an actuator and a collocated PVDF patch which was used as the sensor. Their experimental results demonstrated the effectiveness of the active vibration control method. Direct output feedback-based active vibration control has been implemented on a smart cantilever beam at its resonant frequency using PZT sensors and actuators by Parameswaran, et al., ${ }^{8}$ They compared the performance of the conventional PC-based control and a dedicated real-time control at resonance, and their experimental results demonstrated that the implementation of real-time control provides a much more controlled response of the system with an excellent transient response, as well as a highly reliable steady state response. 
What's more, the acceleration sensor-based control strategy is an effective control method used in vibration suppression. ${ }^{9-11}$ Its best feature is that acceleration is used as the feedback signal, which can be directly measured by accelerometers. In addition, acceleration is easier to measure than strain, displacement, or velocity. ${ }^{9,12}$ Considerable works have been done using the acceleration sensor-based feedback control strategy, showing that acceleration feedback control is effective and robust in vibration measurement and active control. ${ }^{12-17}$

Mahmoodi, et al., ${ }^{9}$ used an active vibration control method called modified acceleration feedback for vibration control of plate structures as an extension of modified positive position feedback. PZT patches were utilized to suppress the vibration of the plate, and the method was successfully verified on a test plate in the laboratory. Preumont, et al., ${ }^{12}$ proposed a local control scheme using acceleration feedback and a collocated proof-mass actuator for active damping of beam-like structures. They conducted experimental investigations of vibration suppression under resonant excitations. Gatti, et al., ${ }^{13}$ conducted a theoretical and experimental study of the active vibration control of a simply supported beam using a piezoelectric patch actuator and a physically collocated accelerometer. Shin, et al., ${ }^{14}$ proposed an active vibration control method of clamped-clamped beams using acceleration feedback controllers with a non-collocated sensor and a moment pair actuator configuration. Qiu, et al., ${ }^{15,16}$ presented an acceleration sensor-based active vibration control for a cantilever beam and a cantilever plate with bonded piezoelectric patches. The phenomenon of phase hysteresis and time delay were considered in their work. The PD control method, a nonlinear control method and acceleration signal-based sliding mode control algorithm were utilized to suppress vibration. Chatterjee ${ }^{17}$ presented a theoretical basis of time-delayed acceleration feedback control of linear and nonlinear vibrations of mechanical oscillators.

In this paper, active vibration control of a doubly-clamped flexible beam with bonded discrete PZT actuators and mounted accelerometer is investigated. The FEM method is utilized to model the system, and the acceleration feedback-based proportional-integral controller and the acceleration feedbackbased variable structure controller are designed. Both theoretical and experimental studies were undertaken to verify the presented methods.

The original contributions of this paper are detailed as follows: (a) The model of a piezoelectric clamped-clamped beam with an accelerometer is obtained by FEM. The FEM model is used for simulations of acceleration feedback control algorithms. (b) Acceleration sensor-based proportional and integral control and variable structure control are designed. (c) Simulations and experiments are conducted to validate the effectiveness of the designed controllers in suppressing the resonant vibration of the clamped-clamped beam under persistent excitation.

This article is organized as follows: In Section 2, the dynamic model of a doubly-clamped piezoelectric beam bonded with piezoelectric actuators and an accelerometer is derived by using the finite element method. In Section 3, the acceleration feedback-based proportional-integral control algorithm and the acceleration feedback based variable structure control method are proposed, and a numerical simulation is carried out. In

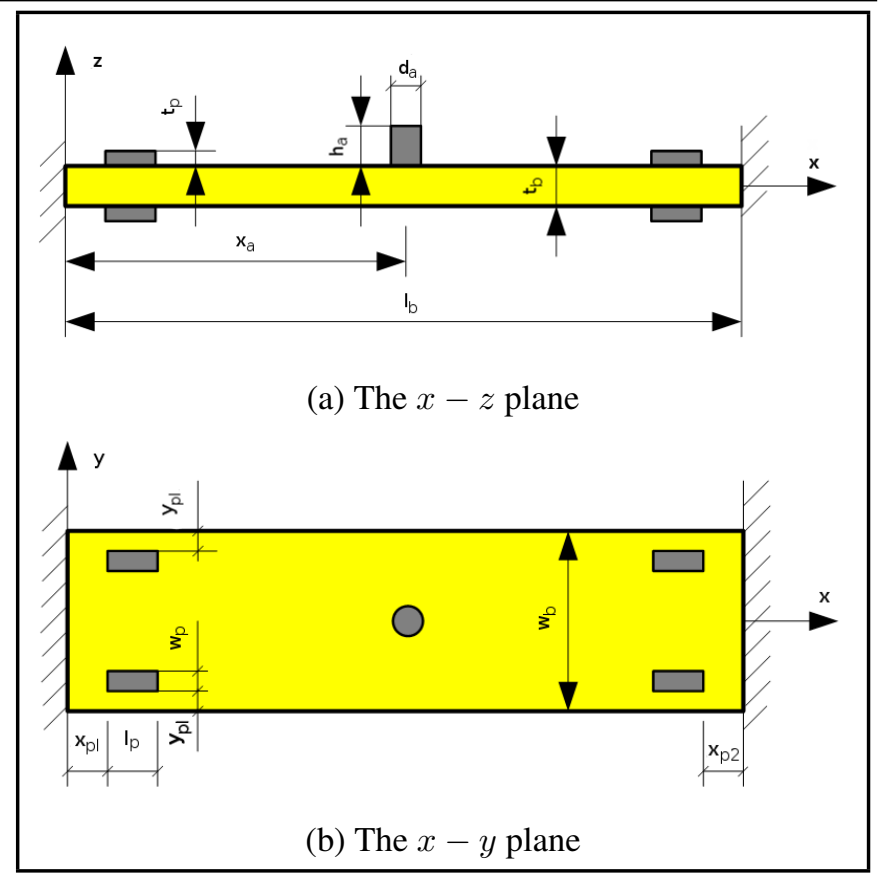

Figure 1. Schematic diagram of a doubly-clamped piezoelectric flexible beam.

Section 4, a doubly-clamped piezoelectric flexible beam experimental system is constructed. Experiments are conducted by using the proposed control algorithms. Finally, the conclusion is drawn in Section 5.

\section{SYSTEM MODELLING}

In this section, a mathematical model is derived for a doubly-clamped piezoelectric beam structure equipped with an acceleration sensor and PZT actuators. The finite element method is used to build the dynamics model. The four-node rectangle plate element is utilized. The state-space representation form of the dynamics model is obtained, including acceleration sensing and piezoelectric driving. Modal analyses are performed.

\subsection{The Doubly-clamped Piezoelectric Flexible Beam}

The doubly-clamped piezoelectric flexible beam is schematically depicted in Fig. 1. The flexible beam is bonded with PZT patches and an accelerometer. Eight PZT patches are bonded on both surfaces of the flexible beam. The patches are divided into two groups, and are used as the excited actuator and control actuator. Each group is composed of four piezoelectric patches, symmetrically bonded on both surfaces close to both clamped sides. Either group can be used as a one-channel actuator by parallel connection. The left group is used as the actuator to excite the vibration of the beam, and the right group is used to suppress the vibration of the beam.

As shown in Fig. 1, the accelerometer is located in the intermediate position of the flexible beam, used to detect the vibration of the beam. The length, width, and thickness of the flexible beam structure are $l_{b}=600 \mathrm{~mm}, w_{b}=120 \mathrm{~mm}$, and $t_{b}=2 \mathrm{~mm}$, respectively. Those of the PZT patches are $l_{p}=50 \mathrm{~mm}, w_{p}=15 \mathrm{~mm}$, and $t_{p}=1 \mathrm{~mm}$, respectively. The diameter and height of the accelerometer are $d_{a}=18 \mathrm{~mm}$ and $h_{a}=22 \mathrm{~mm}$, respectively. The location of the piezoelec- 


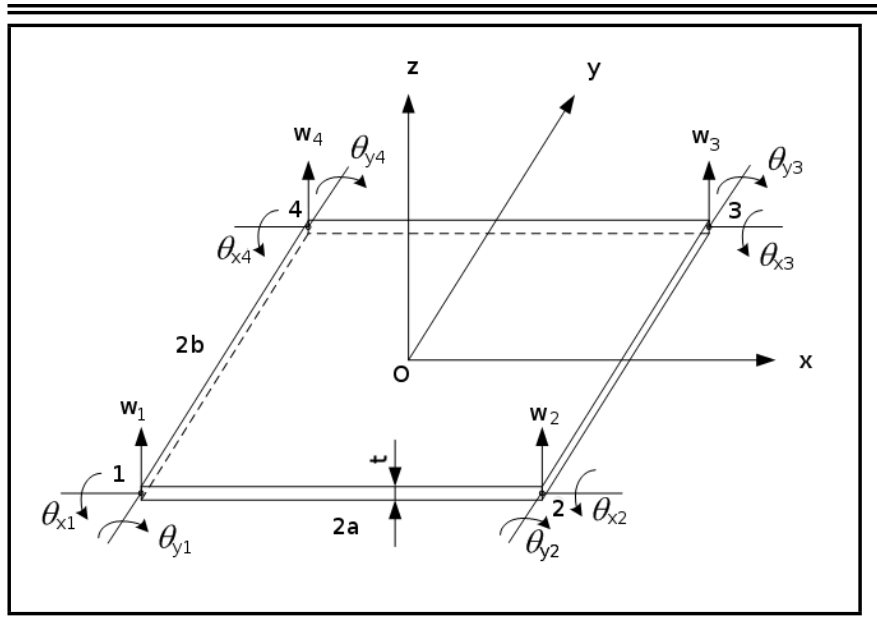

Figure 2. Four-node rectangle plate element.

tric actuators and the accelerometer are shown in Fig. 1, where $x_{p 1}=x_{p 2}=50 \mathrm{~mm}, y_{p 1}=y_{p 2}=15 \mathrm{~mm}$, and $x_{a}=300 \mathrm{~mm}$.

\subsection{The Element Dynamics Equation}

The beam is discretized by using the four-node rectangle plate element, as shown in Fig. 2. There are three degrees of freedom at each node of the four-node rectangle plate element, described as $w, \theta_{x}$, and $\theta_{y}$, respectively. The element nodal displacement vector can be expressed as

$$
\left.\mathbf{d}^{e}=\begin{array}{llllll}
w_{1} & \theta_{x 1} & \theta_{y 1} & w_{2} & \theta_{x 2} & \theta_{y 2} \\
w_{3} & \theta_{x 3} & \theta_{y 3} & w_{4} & \theta_{x 4} & \theta_{y 4}
\end{array}\right]^{T} .
$$

The transverse displacement of any point in the element can be expressed as ${ }^{18}$

$$
w(x, y)=\mathbf{N d}^{e}
$$

where $\mathbf{N} \in \mathbf{R}^{1 \times 12}$ is the displacement shape function.

The element strain matrix can be expressed using the element nodal displacement vector ${ }^{18,19}$

$$
\boldsymbol{\epsilon}=z \mathbf{B d}^{e}
$$

where $\mathbf{B}$ is a matrix representing the relationship between the element strain matrix and element nodal displacement vector, and $z$ is the variable in the thickness direction.

The dynamics equation of the four-node rectangle plate element can be written as ${ }^{20}$

$$
\mathbf{m}_{b}^{e} \ddot{\mathbf{d}}^{e}+\mathbf{k}_{b}^{e} \mathbf{d}^{e}=\mathbf{f}_{e x t}^{e}
$$

where $\mathbf{m}_{b}^{e}$ is the element mass matrix, $\mathbf{k}_{b}^{e}$ is the element stiffness matrix, $\ddot{\mathbf{d}}^{e}$ is the element nodal acceleration vector, $\mathbf{d}^{e}$ is the element nodal displacement vector, and $\mathbf{f}_{e x t}^{e}$ is the element external force vector.

$$
\text { In Eq. (4), } \mathbf{m}_{b}^{e}=\int_{V_{b}} \rho_{b} \mathbf{N}^{T} \mathbf{N} d V, \mathbf{k}_{b}^{e}=\int_{V_{b}} z^{2} \mathbf{B}^{T} \mathbf{D}_{b} \mathbf{B} d V \text {, in }
$$

which $\rho_{b}$ represents the material densities of the flexible beam, $V_{b}$ is the element volumes of the rectangle plate element, $\mathbf{D}_{b}$ is the elastic modulus matrix of the rectangle plate element, $z$ represents the variable in the thickness direction, and its range is $\left[-t_{b} / 2 t_{b} / 2\right]$.

In this investigation, the piezoelectric element is regarded as an ordinary rectangle plate element, so the piezoelectric element mass matrix $\mathbf{m}_{p}^{e}=\int_{V_{p}} \rho_{p} \mathbf{N}^{T} \mathbf{N} d V$, and the piezoelectric element stiffness matrix is $\mathbf{k}_{p}^{e}=\int_{V_{p}} z^{2} \mathbf{B}^{T} \mathbf{D}_{p} \mathbf{B} d V$, in which $\rho_{p}$ denotes the densities of the piezoelectric material, $V_{p}$ is the element volumes of the piezoelectric element, $\mathbf{D}_{p}$ is the elastic modulus matrix of the piezoelectric material, $\mathrm{z}$ represent the variable in the thickness direction, and its range is $\left[-t_{p} / 2 t_{p} / 2\right]$.

As shown in Fig. 1, PZT patches are used as actuators to excite or suppress the vibration of the doubly-clamped piezoelectric beam. When a PZT patch is applied to a voltage only in the thickness direction, the stress matrix is ${ }^{21}$

$$
\boldsymbol{\sigma}=-\mathbf{e}^{T} E_{z}
$$

where $\sigma$ represents the stress matrix in the piezoelectric actuator caused by the electric field, $\mathbf{e}$ is the piezoelectric stress constant vector, and $E_{z}$ is the strength of the electric field within the piezoelectric actuator.

When the input voltage of the piezoelectric actuator is $V_{a}$, the strength of the electric field within the piezoelectric actuator is ${ }^{22}$

$$
E_{z}=\frac{V_{a}}{t_{a}}
$$

where $t_{a}$ is the thickness of the piezoelectric actuator.

Substituting Eq. (6) into Eq. (5), the stress matrix in the piezoelectric actuator is obtained as

$$
\boldsymbol{\sigma}=-\mathbf{e}^{T} \frac{V_{a}}{t_{a}}
$$

The driving force of PZT actuators demands that the stress within the piezoelectric element is uniformly distributed along the thickness direction of the piezoelectric patches. The torque generated by the stress is

$$
\mathbf{M}_{a}=\int_{t_{b} / 2}^{t_{a}+t_{b} / 2} \sigma z d z=-\int_{t_{b} / 2}^{t_{a}+t_{b} / 2} \mathbf{e}^{T} E_{z} d z=\bar{z}_{a} \mathbf{e}^{T} V_{a}
$$

where $\mathbf{M}_{a}$ is the torque caused by the PZT actuator, and $\bar{z}_{a}=$ $\left(t_{b}+t_{a}\right) / 2$ denotes the distance the centreline of the PZT patch to the centreline of the beam.

When a voltage is applied, the work done by the PZT actuator is

$$
\begin{gathered}
\mathbf{F}_{a}^{e} \mathbf{d}^{e}=\iint\left[\begin{array}{lll}
\theta_{x} & \theta_{y} & \theta_{x y}
\end{array}\right] \mathbf{M}_{a} d x d y \\
=\int_{S_{a}} \mathbf{B}^{T} \bar{z}_{a} \mathbf{e}^{T} V_{a} \mathbf{d}^{e} d S=\mathbf{f}_{c t r l}^{e} \cdot V_{a} \mathbf{d}^{e}
\end{gathered}
$$

where $\mathbf{F}_{a}^{e}$ is the equivalent nodal load generated by the PZT actuator, $\mathbf{f}_{c t r l}^{e}$ is the piezoelectric element driving force coefficient vector, $\theta_{x y}$ is the torsional angle corresponding to both the $x$ - and $y$ - axes, and $\mathbf{f}_{c t r l}^{e}=\iint \mathbf{B}^{T} \bar{z}_{a} \mathbf{e}^{T} d x d y$.

\subsection{State-space Form of the System Model}

The element meshing and numbering of nodes and elements for the doubly-clamped beam is shown in Fig. 3. The number in parentheses indicates the element number, and the numbers near the element node indicate the node numbers. There are 24 elements along the length direction and 8 elements along 


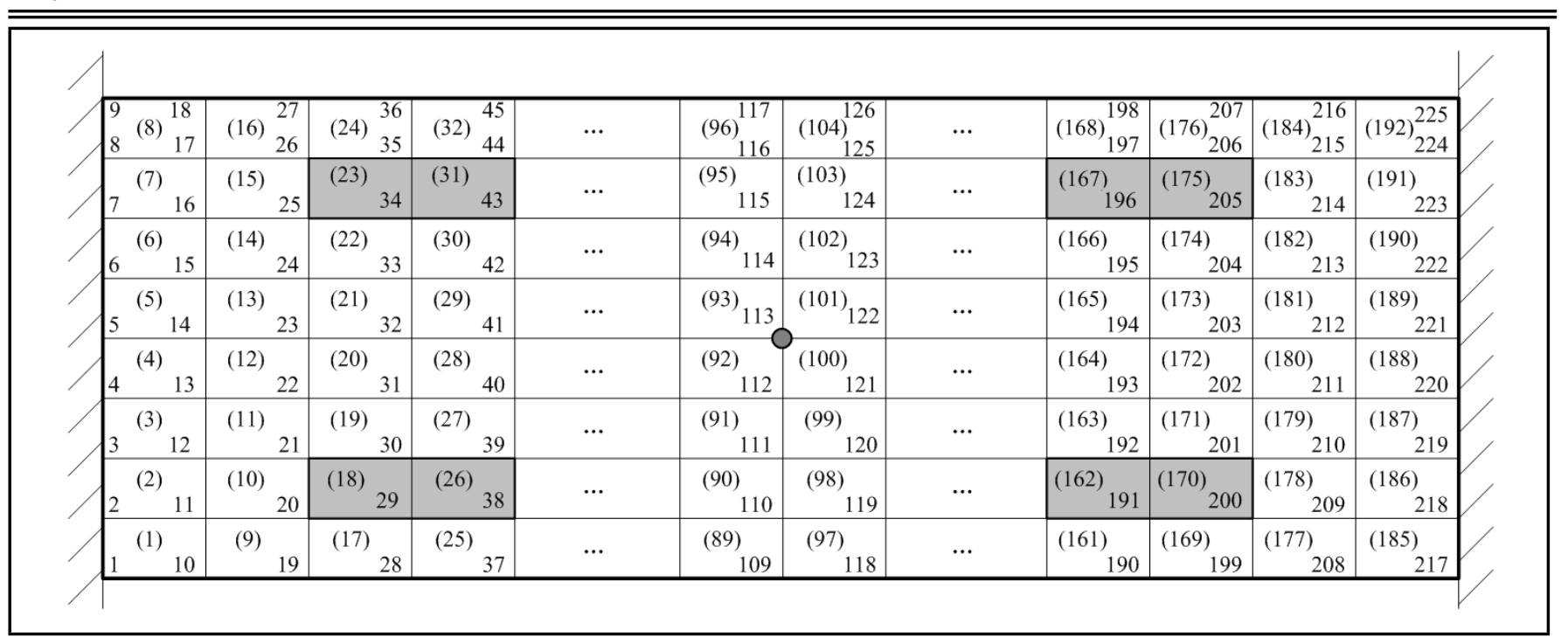

Figure 3. Meshing and numbering of the elements and nodes for the doubly-clamped piezoelectric beam.

the width direction, so the flexible beam is divided into 192 elements and the number of nodes is 225 . The length of the element is $25 \mathrm{~mm}$, and the width of the element is $15 \mathrm{~mm}$. Each of the PZT patches is divided into two parts in the $x$ direction, and one part in the $y$ direction. After applying the boundary condition to the beam, the number of the free nodes of the beam is 207 .

The accelerometer is mounted in the intermediate position of the flexible beam, i.e. the node in the middle position of the beam structure numbered 113 is regarded as the observation node of acceleration output. When the finite element method is used to model the dynamics model of the flexible beam system, the effect of the accelerometer on the structural characteristics should be considered. Here, the effect of the mounted acceleration sensor on the overall stiffness of the structure is ignored; while the mass of the accelerometer cannot be neglected. Its mass is evenly assigned to the four adjacent elements, so the element numbers are 92, 93, 100, and 101.

After assembling the element mass matrix, the element stiffness matrix and the exciting force coefficient vector of the piezoelectric element, one can obtain the global mass matrix, global stiffness matrix and global control force coefficient vector. The boundary conditions of the flexible beam are two clamped sides. Therefore, the corresponding rows and columns should be deleted in the global mass and stiffness matrices, and in the global exciting and control force vectors. Considering the Rayleigh damping effect, the dynamics equation of the piezoelectric flexible beam structure can be written as

$$
\mathbf{M} \ddot{\mathbf{d}}(t)+\mathbf{C} \dot{\mathbf{d}}(t)+\mathbf{K d}(t)=\mathbf{F}_{a} \cdot V_{a}(t)+\mathbf{F}_{c} \cdot V_{c}(t) ;
$$

where $\mathbf{M} \in \mathbf{R}^{3 m \times 3 m}$ and $\mathbf{K} \in \mathbf{R}^{3 m \times 3 m}$ are the global mass matrix and global stiffness matrix, respectively; $\mathbf{C}=$ $\alpha \mathbf{M}+\beta \mathbf{K}$ is the damping matrix, in which $\alpha$ and $\beta$ are Rayleigh damping constants respectively; $\ddot{\mathbf{d}}(t) \in \mathbf{R}^{3 m \times 1}$, $\dot{\mathbf{d}}(t) \in \mathbf{R}^{3 m \times 1}$, and $\mathbf{d}(t) \in \mathbf{R}^{3 m \times 1}$ are the global nodal acceleration vector, the global nodal velocity vector, and global nodal displacement vector, respectively; $\mathbf{F}_{a} \in \mathbf{R}^{3 m \times 1}$ and $\mathbf{F}_{c} \in \mathbf{R}^{3 m \times 1}$ are the global active force vector used to excite the vibration of the flexible beam and the global control force vector that suppresses the vibration of the beam, respectively;
$V_{a}(t)$ is the excitation voltage applied on the vibration excitation piezoelectric actuators; $V_{c}(t)$ is the control voltage applied on the vibration suppression piezoelectric actuators; and $m$ is the total number of nodes, where $m=207$ after applying the boundary conditions.

The output vibration signal measured by the acceleration sensor is ${ }^{12}$

$$
y_{a}(t)=\mathbf{T} \ddot{\mathbf{d}}(t)
$$

where $y_{a}(t)$ is the measured output of the acceleration sensor; $\mathbf{T} \in \mathbf{R}^{1 \times 3 m}$ is the acceleration output matrix corresponding to the observation position; $\ddot{\mathbf{d}}(t)$ is the global nodal acceleration vector; and $m$ is the total number of the nodes.

For the convenience of numerical simulation, Eqs. (10) and (11) should be converted into a state-space form. By using a modal transformation $\mathbf{d}=\mathbf{\Phi g}$, the dynamics equations of the system can be written as ${ }^{23}$

$$
\overline{\mathbf{M}} \ddot{\mathbf{g}}(t)+\overline{\mathbf{C}} \mathbf{g}(t)+\overline{\mathbf{K}} \mathbf{g}(t)=\overline{\mathbf{F}}_{a} \cdot V_{a}(t)+\overline{\mathbf{F}}_{c} \cdot V_{c}(t)
$$

and

$$
y_{a}(t)=\mathbf{T} \boldsymbol{\Phi} \ddot{\mathbf{g}}(t)
$$

where $\boldsymbol{\Phi}$ is the modal matrix; $\mathrm{g}$ is the modal coordinate vector, $\overline{\mathbf{M}}=\boldsymbol{\Phi}^{T} \mathbf{M} \boldsymbol{\Phi}$ is the generalized mass matrix; $\overline{\mathbf{C}}=\boldsymbol{\Phi}^{T} \mathbf{C \Phi}$ is the generalized damping matrix; $\overline{\mathbf{K}}=\boldsymbol{\Phi}^{T} \mathbf{K} \boldsymbol{\Phi}$ is the generalized stiffness matrix; $\overline{\mathbf{F}}_{a}$ is the generalized vibration excitation force vector; and $\overline{\mathbf{F}}_{c}$ is the generalized vibration control force vector.

The state-space representation of Eqs. (12) and (13) is expressed as

$$
\left\{\begin{array}{l}
\dot{\mathbf{X}}(t)=\mathbf{A X}(t)+\mathbf{B}_{a} V_{a}(t)+\mathbf{B}_{c} V_{c}(t) \\
y_{a}(t)=\mathbf{C X}(t)+\mathbf{D}_{a} V_{a}(t)+\mathbf{D}_{c} V_{c}(t)
\end{array}\right.
$$

where $\mathbf{X}=[\mathbf{g}(t) \dot{\mathbf{g}}(t)]^{T}$ is the state vector; $\mathbf{A}=$ $\left[-\overline{\mathbf{M}}^{-1} \overline{\mathbf{K}}-\overline{\mathbf{M}}^{-1} \overline{\mathbf{C}}\right]$ is the system matrix, $\mathbf{B}_{a}=\left[\begin{array}{c}\mathbf{0} \\ \overline{\mathbf{M}}^{-1} \mathbf{\Phi}^{T} \mathbf{F}_{a}\end{array}\right]$ is the vibration excitation force matrix; $\mathbf{B}_{c}=\left[\begin{array}{c}\overline{\mathbf{M}}^{-1} \mathbf{\Phi}^{T} \mathbf{F}_{c} \\ \text { is }\end{array}\right.$ the vibration control force matrix; $\mathbf{C}=-\mathbf{T} \boldsymbol{\Phi}\left[\overline{\mathbf{M}}^{-1} \overline{\mathbf{K}} \overline{\mathbf{M}}^{-1} \overline{\mathbf{C}}\right]$ is the acceleration output matrix; and $\mathbf{D}_{a}=\mathbf{T} \boldsymbol{\Phi} \overline{\mathbf{M}}^{-1} \boldsymbol{\Phi}^{T} \mathbf{F}_{a}$ and $\mathbf{D}_{c}=\mathbf{T} \boldsymbol{\Phi} \overline{\mathbf{M}}^{-1} \boldsymbol{\Phi}^{T} \mathbf{F}_{c}$ are direct transfer coefficients. 
Table 1. The modal frequencies of the first four modes of the doubly-clamped beam.

\begin{tabular}{||c|c|c|c|}
\hline Order & $\begin{array}{c}\text { ANSYS software } \\
(\mathrm{Hz})\end{array}$ & $\begin{array}{c}\text { FEM model } \\
(\mathrm{Hz})\end{array}$ & Relative error \\
\hline 1 & 21.453 & 21.8914 & $2.04 \%$ \\
2 & 67.212 & 66.7915 & $-0.65 \%$ \\
3 & 81.231 & 81.3994 & $0.21 \%$ \\
4 & 116.33 & 116.6680 & $0.32 \%$ \\
\hline
\end{tabular}

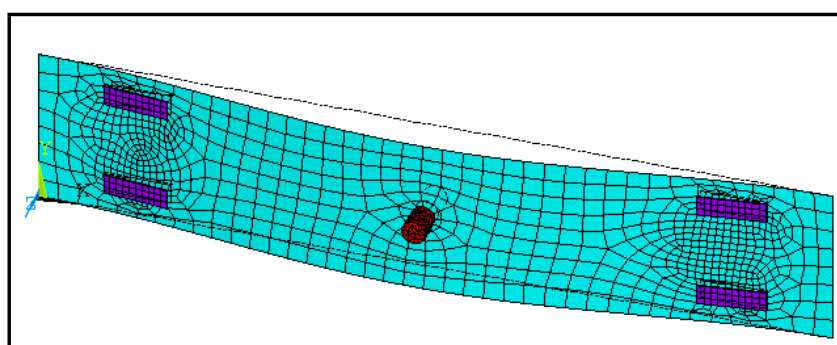

(a) The first mode

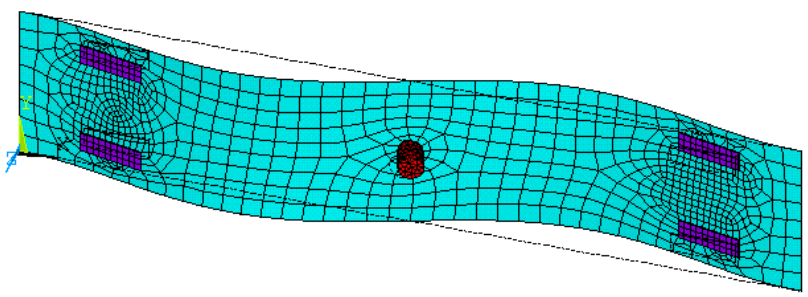

(b) The second mode

Figure 4. The first four vibration modes of the doubly-clamped beam obtained by employing ANSYS software.

\subsection{Modal Analysis of the System}

The geometrical size of the beam, the PZT patch, and the accelerometer can be seen in section 2.1. The flexible beam is made of epoxy resin. Young's modulus, Poisson's ratio, and the mass density of the beam structure are $E_{b}=34.64 \mathrm{Gpa}, \nu_{b}=0.33$ and $\rho_{b}=1865 \mathrm{Kg} / \mathrm{m}^{3}$, respectively. Young's modulus, Poisson's ratio, and the mass density of the PZT patches are $E_{p}=63 \mathrm{Gpa}, \nu_{p}=0.33$, and $\rho_{p}=7650 \mathrm{Kg} / \mathrm{m}^{3}$, respectively, and the piezoelectric strain constant is $d_{31}=166 \times 10^{-12}$. The mass of the accelerometer is $38 \mathrm{~g}$, and Young's modulus, Poisson's ratio, and the mass density are $E_{b}=210 \mathrm{Gpa}, \nu_{b}=0.3$ and $\rho_{b}=7850 \mathrm{Kg} / \mathrm{m}^{3}$, respectively.

The model of the doubly-clamped beam system can be obtained by using the finite element modelling method, and one can also get modal frequencies and modal shapes of the system by using ANSYS software. When ANSYS software is employed to carry out modal analyses, the SHELL63 element is used to generate mesh of the beam, and the SOLID45 element is used to generate mesh of the PZT patches and the accelerometer. The modal frequencies of the flexible beam system are listed in Table 1, calculated by using the FEM method and ANSYS, respectively. The relative errors between the two methods are less than $3 \%$. Comparison of the results demonstrates the correctness of the model built by using the FEM. Figures 4 (a) and (b) illustrate the mode shapes of the first two modes obtained by ANSYS.

\section{CONTROL ALGORITHM AND NUMERICAL SIMULATION}

\subsection{Acceleration Feedback-based Proportional-Integral Control}

In order to suppress the vibration under resonant excitation of a doubly-clamped beam effectively, active control algorithms need to be designed. During this investigation, the system output is the acceleration in the middle of the beam measured by the accelerometer. The measured signals of acceleration sensors generally comprise a large amount of noises, and the derivative of such signals will cause a much larger amount of noises. Since the integration of the measured acceleration signals will attenuate the measured noises, the acceleration sensor-based proportional-integral feedback control algorithm is utilized. To filter out the high frequency noises, a band-pass filter is applied to process the signals measured by the acceleration sensor before the controller design. The central frequency of the designed filter is $21.8914 \mathrm{~Hz}$, and its bandwidth is $30 \mathrm{rad} / \mathrm{s}$ in the numerical simulation.

The acceleration sensor-based proportional-integral feedback control algorithm is

$$
u(k)=-K_{p} y_{a c c}(k)-K_{I} \sum_{j=1}^{k} y_{a c c}(j) ;
$$

where $k$ is the serial number of the sampling time points; $y_{a c c}(k)$ is the acceleration signal at the sampling instants; $u(k)$ is the control output; $K_{P}$ is the proportional gain; and $K_{I}$ is the integral gain.

\subsection{Acceleration Feedback-based Variable Structure Control}

The variable structure control (VSC) algorithm is easily implemented, including selecting the switching function and the control law. ${ }^{24,25}$ When sliding mode variable structure control methods are designed to suppress the vibration of the doublyclamped beam, the switching function of the VSC is composed of both the acceleration signal and its integration. The switching function is expressed as

$$
s(k)=-c_{1} y_{a c c}(k)-c_{2} \sum_{j=0}^{k} y_{a c c}(j) ;
$$

where $y_{a c c}(k)$ is the acceleration signal at the sampling instants, and $c_{1}$ and $c_{2}$ are constants greater than 0 .

When the switch variable structure control is designed, the switching function at the sampling interval is selected as a switch to determine the controller's output. Therefore, the acceleration-based switch variable structure control law $u_{v}(k)$ is written as

$$
u_{v}(k)=\operatorname{Asgn}[s(k)] ;
$$

where $A$ is a positive constant, denoting the control amplitude; $\operatorname{sgn}(\cdot)$ is the sign function; and it is

$$
\operatorname{sgn}[s(k)]=\left\{\begin{array}{cc}
1, & s(k) \geq 0 \\
-1, & s(k)<0
\end{array} .\right.
$$

However, to realize the switch variable structure control, VSC requires high switching frequency associated with large 


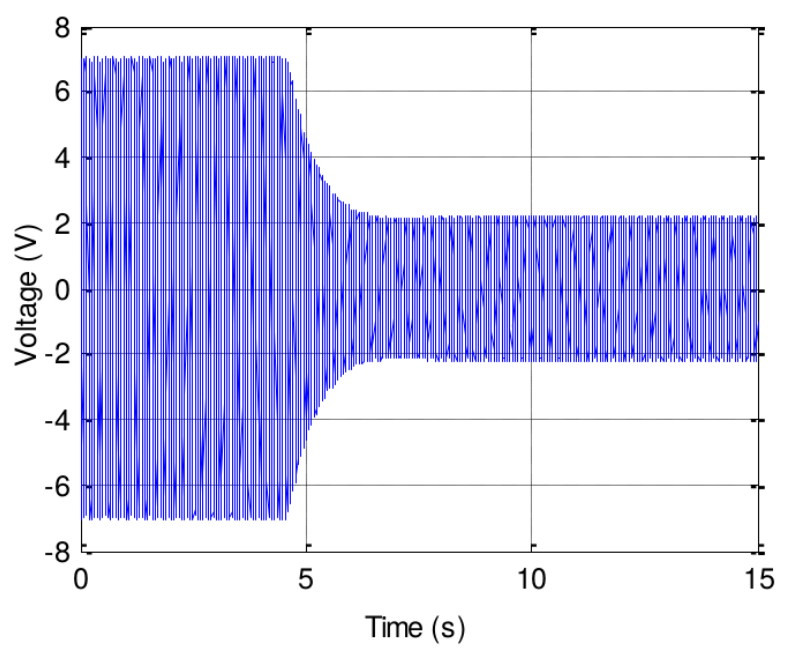

(a) Resonant vibration before and after control

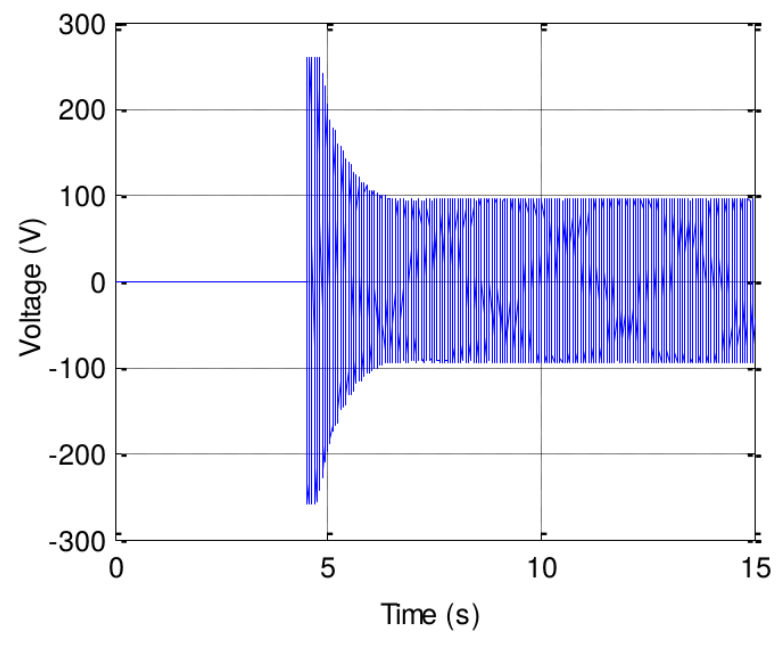

(c) Control voltage

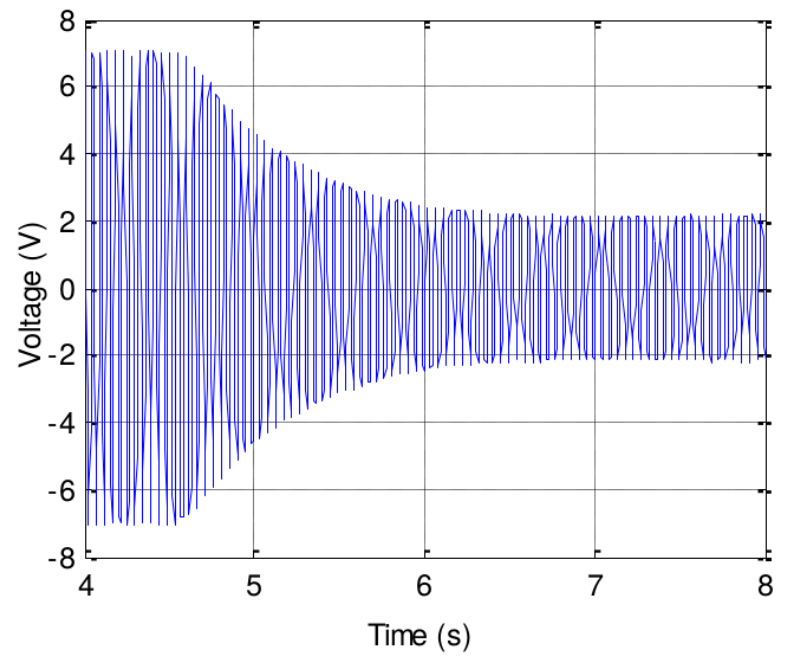

(b) Zoom in on the time axis of Fig. 5 (a)

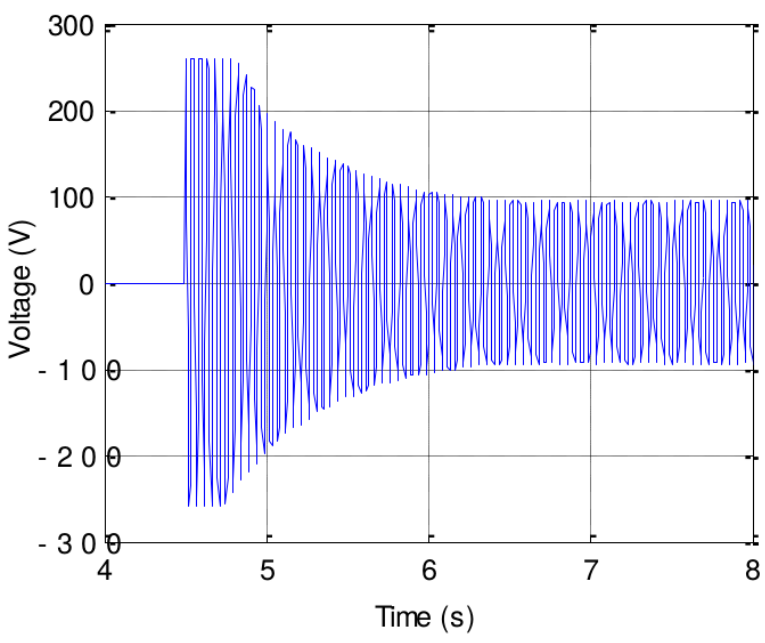

(d) Zoom in on the time axis of Fig. 5 (a)

Figure 5. Simulation results of acceleration based proportional-integral control.

control gains, which in practice may cause chattering and excite the high frequency vibration of the system. To avoid the problem of this switching method, the switch surface $s_{l a w}(k)$ is selected as

$$
s_{\text {law }}(k)=-\epsilon \operatorname{sgn}[s(k)]-q s(k) ;
$$

where $s(k)$ is the switching function mentioned above, and $\epsilon$ and $q$ are positive constants.

Then, the control law of the improved VSC $u_{r}(k)$ is

$$
u_{r}(k)=\operatorname{Asgn}\left[s_{\text {law }}(k)\right] \text {. }
$$

\subsection{Numerical Simulation Results}

In this section, numerical simulations are carried out to evaluate the effectiveness of the algorithms designed in section 3.1 and section 3.2. The structural size and mechanical properties of the flexible beam, the PZT patches and the accelerometer are described in the previous section. By using the FEM method, the matrices corresponding to the first two modes model are obtained as $\mathbf{A}=$

$$
\begin{aligned}
& {\left[\begin{array}{cccc}
0 & 0 & 1 & 0 \\
0 & 0 & 0 & 1 \\
-1.8919 \times 10^{4} & 1.1126 \times 10^{-6} & -1.2379 & 0 \\
1.1726 \times 10^{-6} & -1.1612 \times 10^{5} & 0 & -2.5180
\end{array}\right],} \\
& \mathbf{B}_{a}=\left[\begin{array}{c}
0 \\
0 \\
0.0042 \\
0.0012
\end{array}\right], \quad \mathbf{B}_{c}=\left[\begin{array}{c}
0 \\
0 \\
0.0042 \\
-0.0012
\end{array}\right], \quad \mathbf{C}= \\
& {\left[\begin{array}{ll}
4.99 \times 10^{4}-8.91 \times 10^{-6} & 3.2715 \\
0
\end{array}\right], D_{a}=-0.011,} \\
& D_{c}=-0.011 .
\end{aligned}
$$

In the simulation research, the sampling period is specified as $T_{s}=3 \mathrm{~ms}$. By converting the continuous-time model to the discrete-time one, the coefficient matrices of the discretetime system are obtained. The numerical simulation can be carried out by using the coefficient matrices of the discretetime system. The excited signal actuated on the actuators is a sinusoidal signal, and the frequency of the excited signal is $21.8914 \mathrm{~Hz}$, with an amplitude of $130 \mathrm{~V}$.

The control gains of the acceleration sensor-based proportional-integral feedback control algorithm are chosen as $K_{p}=1.0, K_{I}=0.01$. The control action is applied at the 


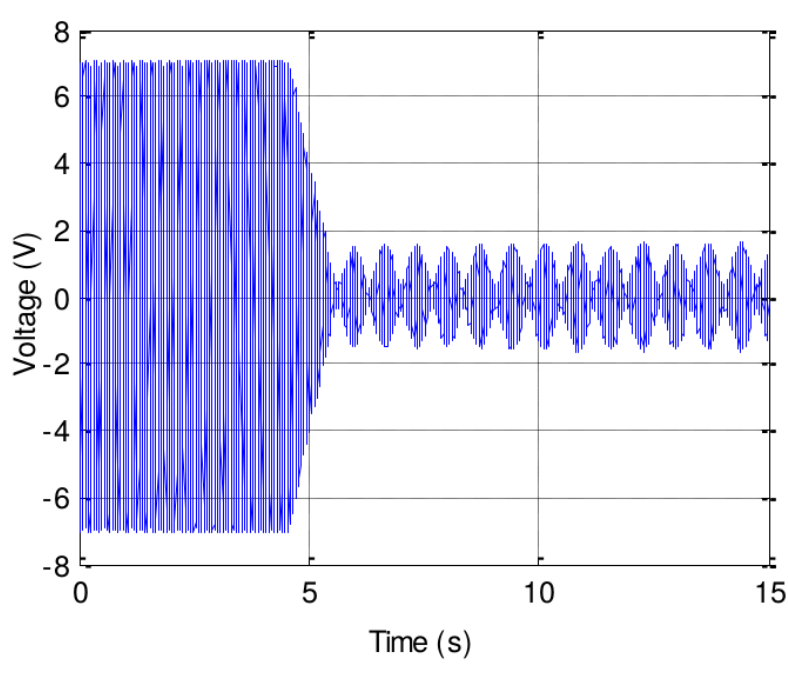

(a) Resonant vibration before and after control

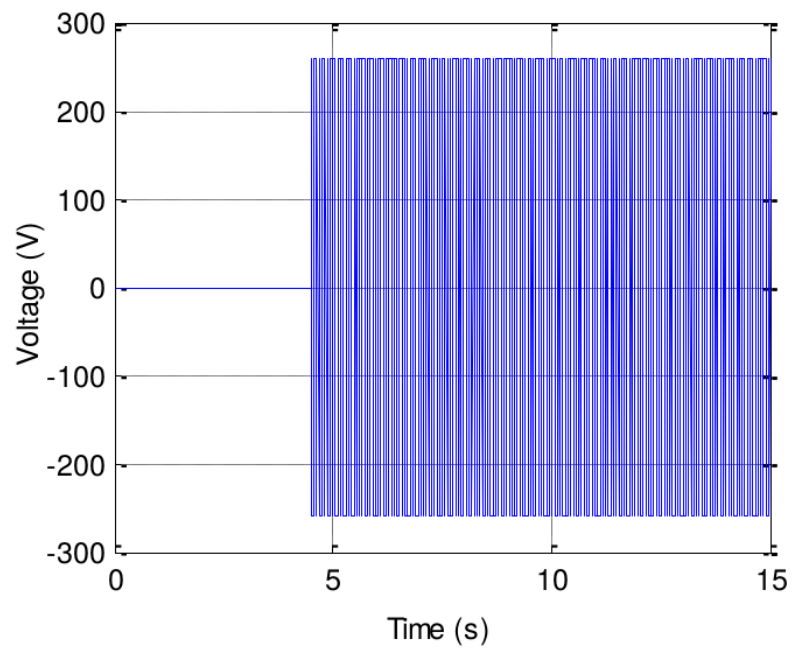

(c) Control voltage

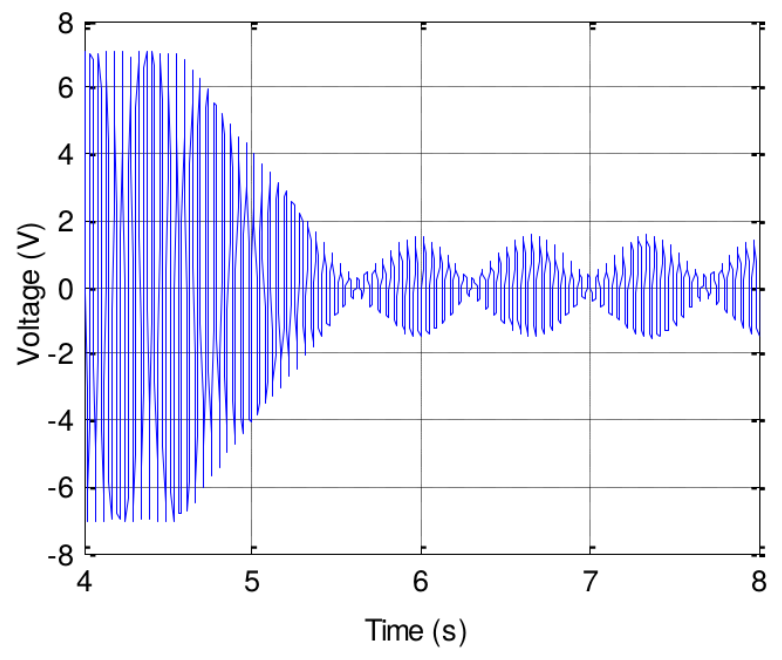

(b) Zoom in on the time axis of Fig. 6 (a)

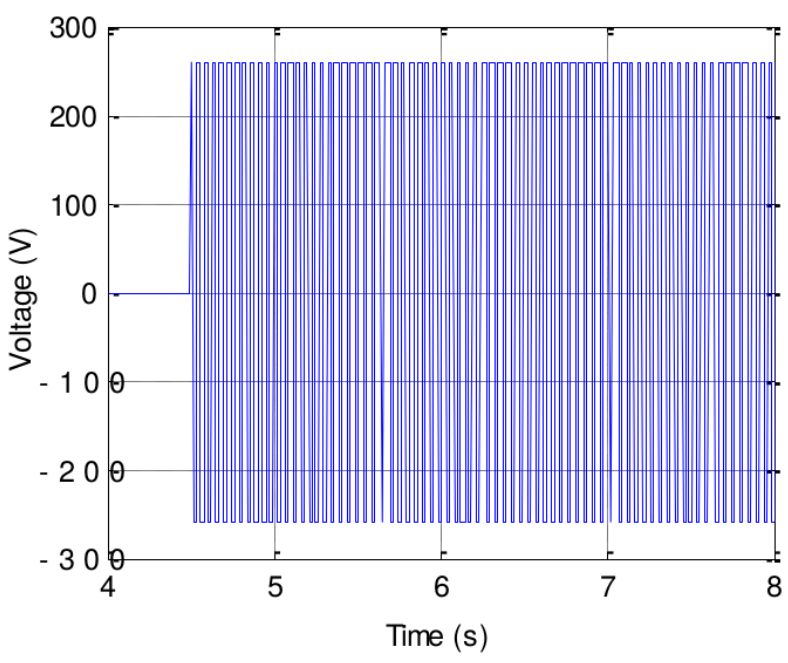

(d) Zoom in on the time axis of Fig. 6 (c)

Figure 6. Simulation results of switch variable structure control.

moment of $t=4.5 \mathrm{~s}$. The time-domain resonant vibration response before and after control is shown in Fig. 5 (a), and the control voltage applied on the PZT actuators is plotted in Fig. 5 (c). Figures 5 (b) and (d) are the enlarged view of Figs. 5 (a) and (c) from 4 seconds to 8 seconds, respectively. As depicted in Figs. 5 (a) and (b), when the control action is not applied, the vibration amplitude is about $7 \mathrm{~V}$. After applying the active control, the vibration is suppressed, and the vibration amplitude will gradually become smaller. When the vibration response is stable, the vibration amplitude is about $2.2 \mathrm{~V}$. As depicted in Fig. 5 (c), when the control action is not applied, the control voltage is $0 \mathrm{~V}$. When the active control is applied, the control voltage reaches saturation in a short time, and then decreases in stable amplitude. From the simulation results, it can be seen that the amplitude of the control voltage and the controlled vibration response can stay at a constant level when applying the proportional plus integral control algorithm.

When using the switch variable structure control algorithm, the control gain is specified as $A=5$, and the constants are selected as $c_{1}=0.65, c_{2}=0.01$. Figure 6 (a) shows the timedomain resonant vibration response before and after control.
Figure 6 (c) shows the control voltage applied on the PZT actuators. Figures 6 (b) and (d) are the enlarged view of Figs. 6 (a) and (c) from 4 seconds to 8 seconds, respectively. When the control action is not applied, the vibration amplitude is about $7 \mathrm{~V}$. When the active control is applied, the vibration is suppressed. When the vibration response is stable, the vibration amplitude increases and decreases periodically; the maximum vibration amplitude is about 1.5 V. As depicted in Figs. 6 (c) and (d), the control voltage is saturated continuously at a high frequency switching once the active control is applied.

When using the improved variable structure control algorithm, the parameters are chosen as $A=5, c_{1}=0.7$, $c_{2}=0.02, \epsilon=2.5$, and $q=1.2$. Fig. 7 (a) shows the time-domain resonant vibration response before and after control. Figure 7 (c) shows the control voltage applied on the PZT actuators. Figures 7 (b) and (d) are the enlarged view of Figs. 7 (a) 7 (c) from 4 seconds to 8 seconds, respectively. When the active control is applied, the vibration is suppressed significantly. When the vibration is stable, the vibration amplitude increases and decreases periodically, and the maximum vibration amplitude is about 0.9 V. As depicted in Fig. 7 (c), 


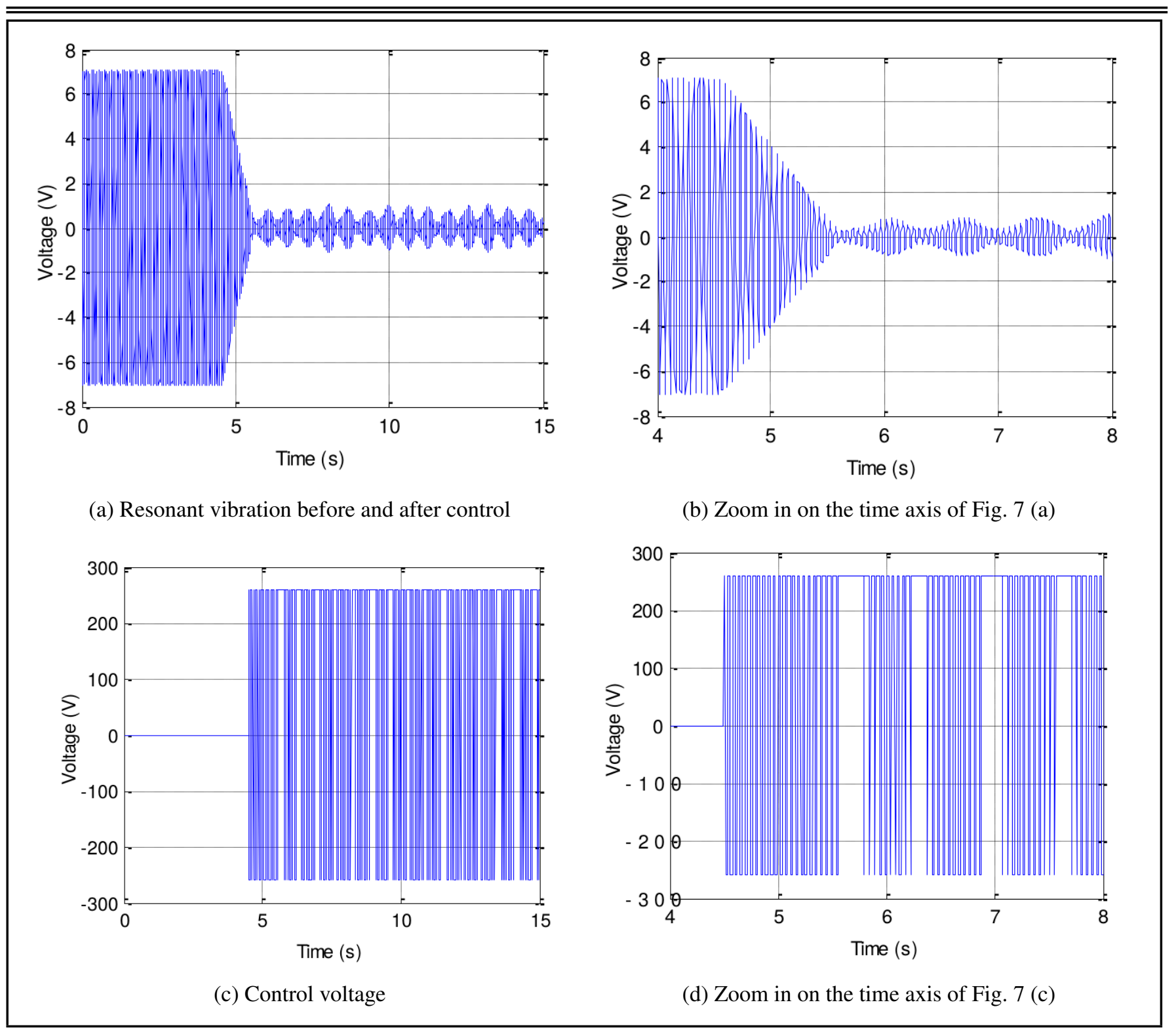

Figure 7. Simulation results of improved variable structure control.

once the active control is applied, the control voltage is saturated continuously. The reason that plots 7 (c) and (d) have several intervals is that a building time is needed; thus, the switch does not happen.

From Figs. 6 and 7, one can see that the control voltage amplitude of the variable structure control is the maximum value. This can provide the maximum control energy to suppress the periodical vibration. The vibration response amplitudes change periodically. This is because the following reasons: if the vibration amplitude is suppressed to become much smaller, the control value stays for a smaller time. Then the vibration amplitude will be increased. After the vibration becomes larger, the control effects are applied rapidly. Thus, the vibration amplitude will decrease.

From the simulation results, one can see that the acceleration feedback-based control algorithm can suppress the resonant vibration effectively. The amplitudes of resonant vibrations are reduced much better than with PI control, as compared to those of the designed variable control algorithms. Furthermore, the improved variable structure control algorithm shows the best control performance among the three methods.

\section{EXPERIMENTS}

In order to verify the effectiveness of the designed acceleration sensor based vibration control method, a doubly-clamped piezoelectric flexible beam experimental system was developed. Experiments on modal identification and resonant vibration suppression of the piezoelectric flexible beam were conducted.

\subsection{Introduction of the Experimental Setup}

The schematic diagram of the doubly-clamped piezoelectric beam experimental system is illustrated in Fig. 8. The experimental setup constitutes a doubly-clamped flexible beam structure bond with PZT patches bonded on both sides, and an accelerometer (type: CA-YD-127) is mounted in the intermediate position of the beam.

The photograph of the experimental setup is shown in Fig. 9. The practical locations of the PZT patches and the accelerometer sensor are schematically illustrated in Fig. 1. The dimensions and material properties of the beam, piezoelectric patches, and the accelerometer are given in Section 2.4. A 


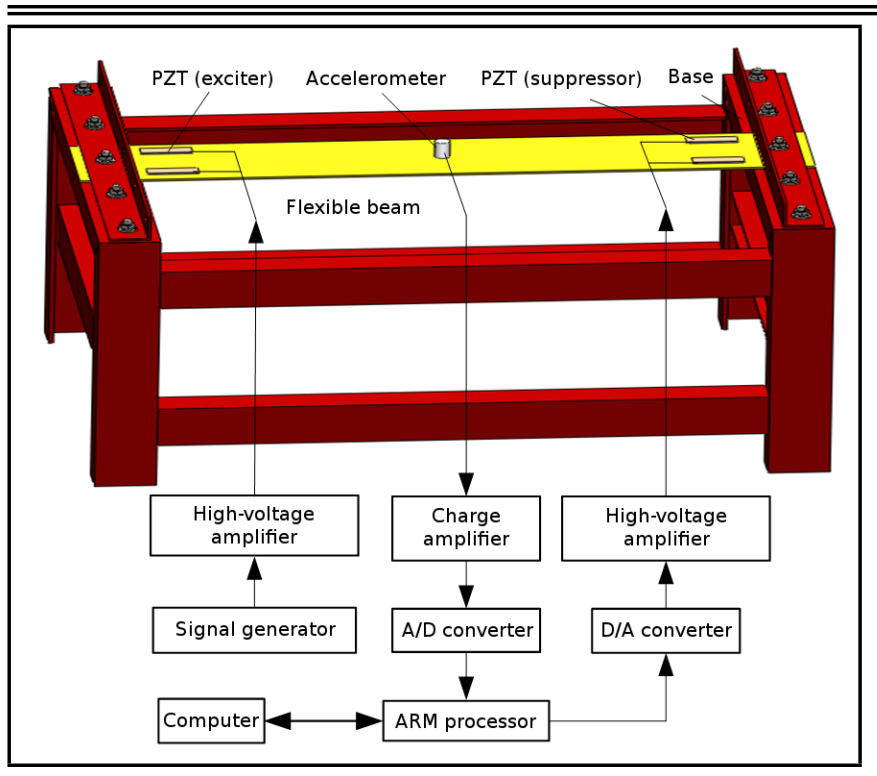

Figure 8. Schematic diagram of the doubly-clamped piezoelectric beam experiment system.

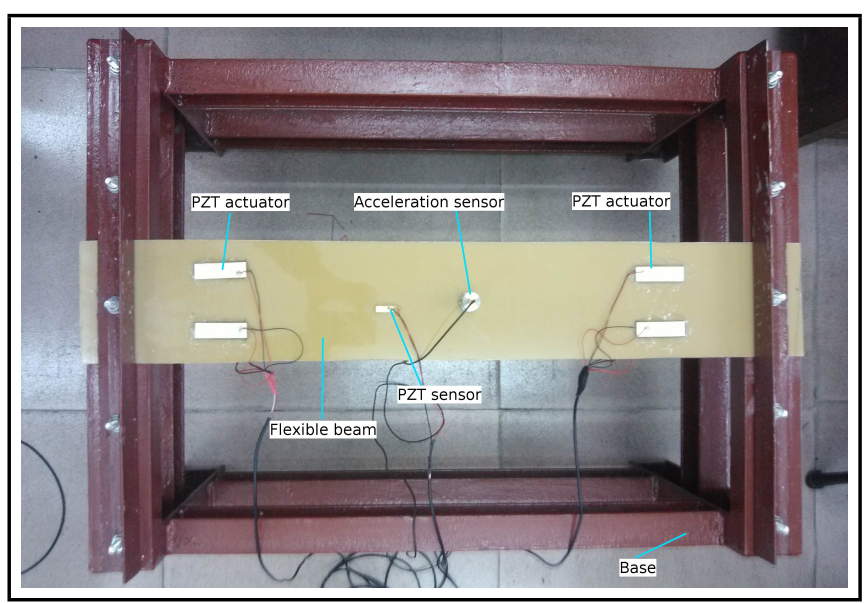

Figure 9. Experimental setup of the doubly-clamped beam.

signal generator (type: SP-F05) is used to generate sinusoidal excitation signals. The generated excitation signal is amplified by a piezoelectric amplifier (APEX PA240CX) to excite the resonant vibration of the beam by using the excited piezoelectric actuator. The piezoelectric amplifier used for vibration excitation can amplify the excitation sinusoidal signal from a low voltage range, from $-5 \mathrm{~V}$ to $+5 \mathrm{~V}$ to a high voltage range, from $-130 \mathrm{~V}$ to $+130 \mathrm{~V}$. The piezoelectric acceleration sensor's signal is amplified by charge amplifiers (YE5850) to the voltage range of $-10 \mathrm{~V}$ to $+10 \mathrm{~V}$, and converted into digital data through an A/D (analog to digital) chip (AD7862, 4-channel, $12 \mathrm{Bit}$ ). The output of the controller is sent to the piezoelectric amplifier used for vibration suppression through a D/A (digital to analog) converter (AD7847, 2-channel 12-bit). The vibration suppression piezoelectric actuators are driven by a highvoltage amplifier APEX PA241DW, which amplifies the signal from a low voltage range, from $-5 \mathrm{~V}$ to $+5 \mathrm{~V}$, to high voltage range, from $-260 \mathrm{~V}$ to $+260 \mathrm{~V})$. An ARM board keeps communicating with a personal computer (PC), which is used as the signal processing and control system. The sampling period of the control experiments is selected as $3 \mathrm{~ms}$.
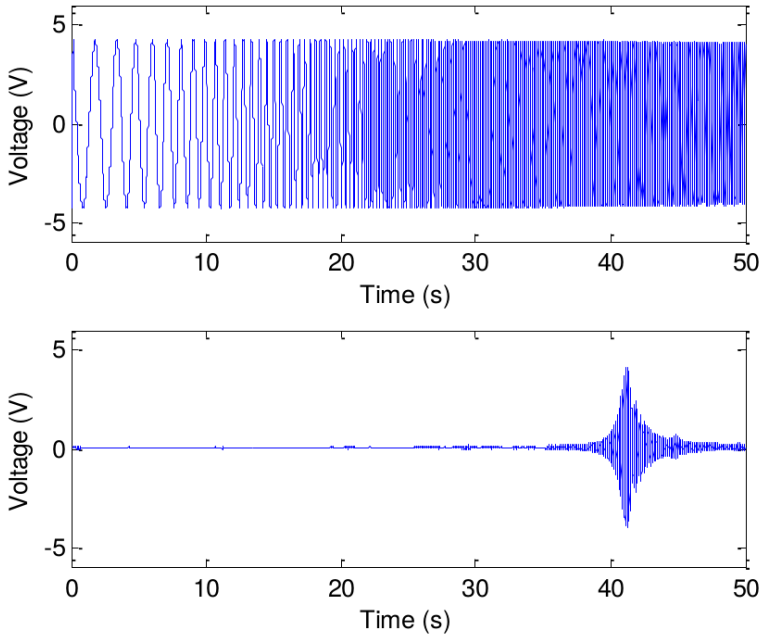

(a) The excited swept sine signal and time-domain response of the flexible beam.

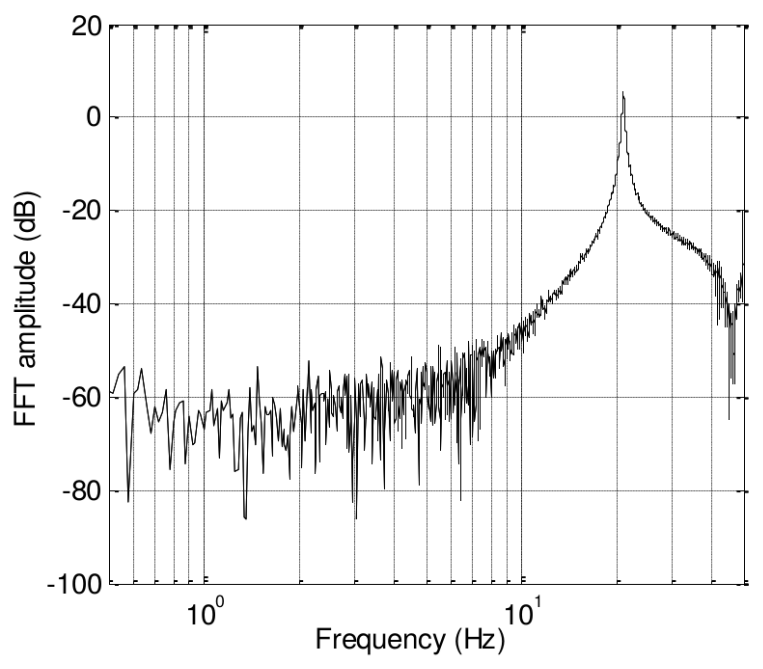

(b) Frequency response

Figure 10. Acceleration sensor measured swept sine vibration response excited by PZT actuators.

\subsection{Modal Frequency Identification of the Flexible Beam}

To identify the practical modal frequencies of the doublyclamped piezoelectric beam, excitation analyses are used. The swept sine signal is generated by a signal generator SP-F05, ranging from $0.5 \mathrm{~Hz}$ to $50 \mathrm{~Hz}$, and the exciting time and amplitude is $50 \mathrm{~s}$ and $4 \mathrm{~V}$, respectively. Then, it is amplified by a high-voltage amplifier APEX PA240CX and applied to the vibration excitation PZT actuators. Thus, the swept frequency response is obtained. Figure 10 (a) shows the excited swept sinusoidal signal and the time-domain response signal measured by the piezoelectric acceleration sensor. By employing fast Fourier transform (FFT), one can obtain the frequency response as shown in Fig. 10 (b). From Fig. 10 (b), it can be known that the first modal frequency of the doubly-clamped beam is $21.0 \mathrm{~Hz}$.

In practice, the measured signals of acceleration sensors generally comprise a large amount of noises when the vibration of the first mode of the doubly-clamped beam is excited 


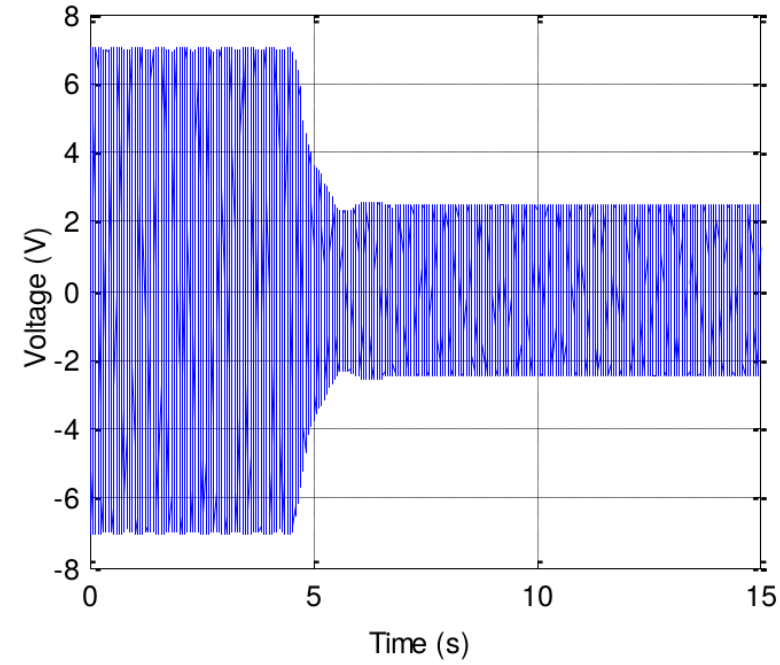

(a) Resonant vibration before and after control

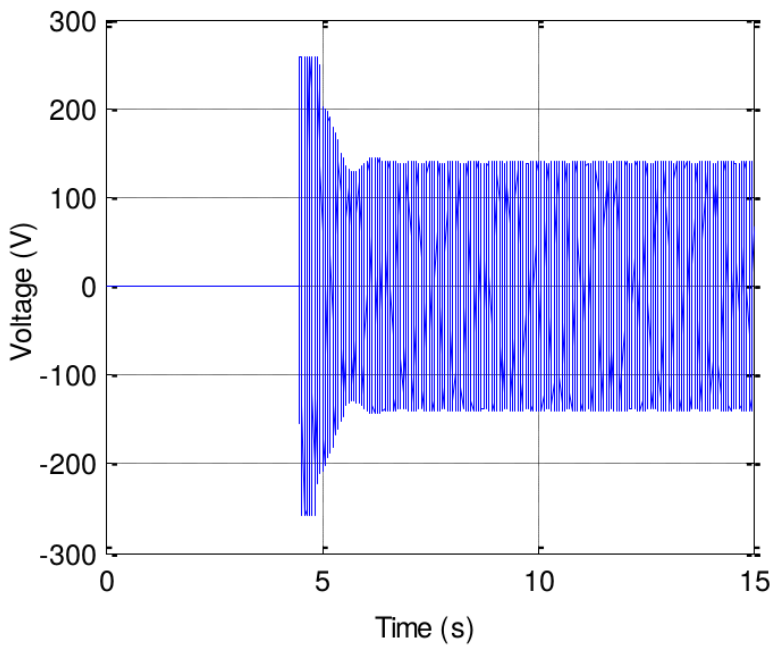

(c) Control voltage

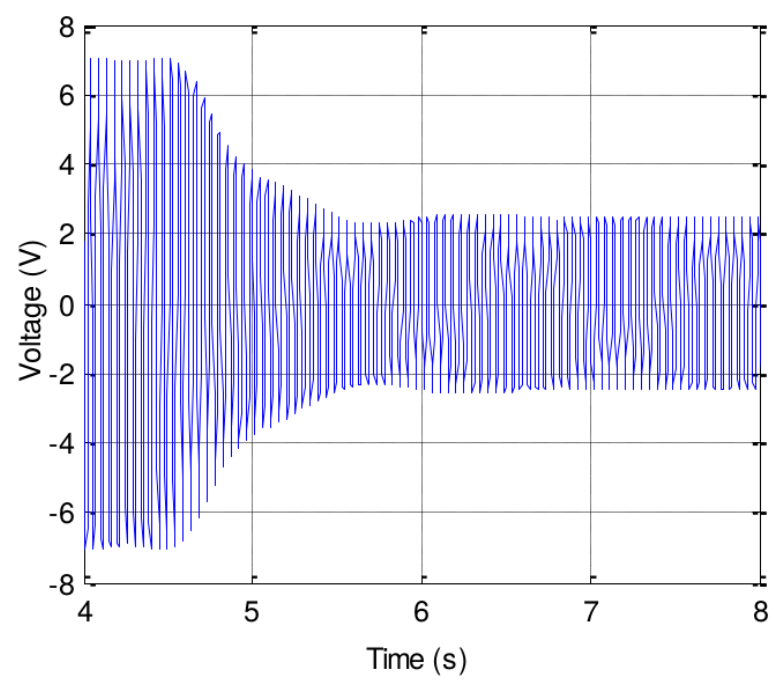

(b) Zoom in on the time axis of Fig. 11 (a)

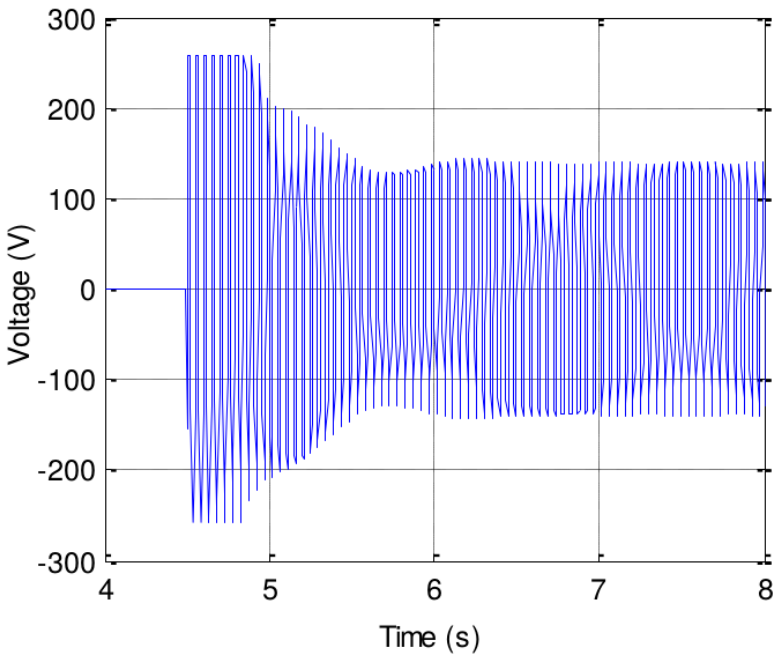

(d) Zoom in on the time axis of Fig. 11 (c)

Figure 11. Experimental result of acceleration-based proportional-integral control.

by PZT actuators. To filter out the high frequency noises of the measured signal, a band-pass filter must be applied to controller design, and the central frequency of the filter is $21.0 \mathrm{~Hz}$ and its bandwidth is $30 \mathrm{rad} / \mathrm{s}$ in the experimental investigation.

\subsection{Experiment Results on Resonant Vibration Suppression}

For resonant vibration suppression of the first vibration mode, the sinusoidal excitation signal is generated by the signal generator SP-F05. The amplitude and frequency of the sinusoidal excitation signal are $4 \mathrm{~V}$ and $21.0 \mathrm{~Hz}$, respectively. The frequency is the same as that of the first vibration mode. Firstly, the acceleration sensor-based proportionalintegral feedback control method is used to suppress the vibration under resonant excitation.

The control gains of the acceleration sensor-based proportional-integral feedback control algorithms are specified as $K_{p}=1.1, K_{I}=0.02$. The control parameters between simulations and experiments are a little different. This is the reason why the parameters used in the simulation are not precisely consistent with those of the flexible physical beam, which was used perfectly in the experimental material. The physical parameters of the flexible plate, such as the material density, Young's modulus, uniformity, etc., cannot be precisely calculated. Moreover, the mass of the glue and the connected signal wires of the PZT patches are not considered in the model. These factors will affect the characteristics of the system. Therefore, the control parameters are inconsistently selected.

The control action is applied at the moment of $t=4.5 \mathrm{~s}$. The time-domain resonant vibration responses before and after control and control voltage are applied on the PZT actuators are shown in Figs. 11 (a) and (c), respectively. Figures 11 (b) and (d) are the enlarged view of Figs. 11 (a) and (c) from 4 seconds to 8 seconds. As depicted in Fig. 11 (a), when the control action is not applied, the vibration amplitude is about $7 \mathrm{~V}$. After the active control is applied, the control voltage reaches saturation abruptly. The beam's large amplitude vibration is suppressed to a small amplitude vibration quickly, and the control voltage is decreased accordingly. When the vibration is stable, the vibration amplitude is about $2.5 \mathrm{~V}$, and the amplitude of the control voltage is almost unchanged. 


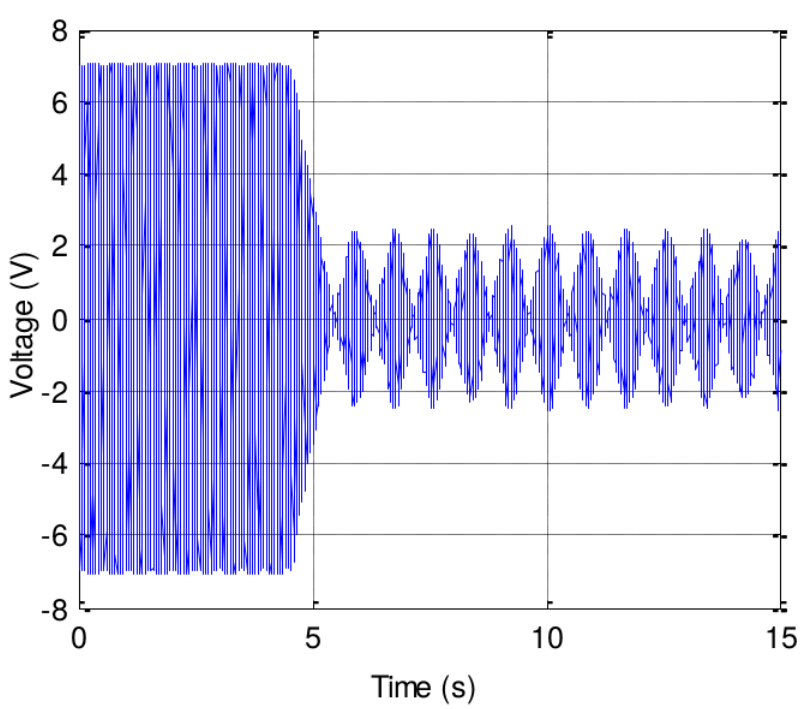

(a) Resonant vibration before and after control

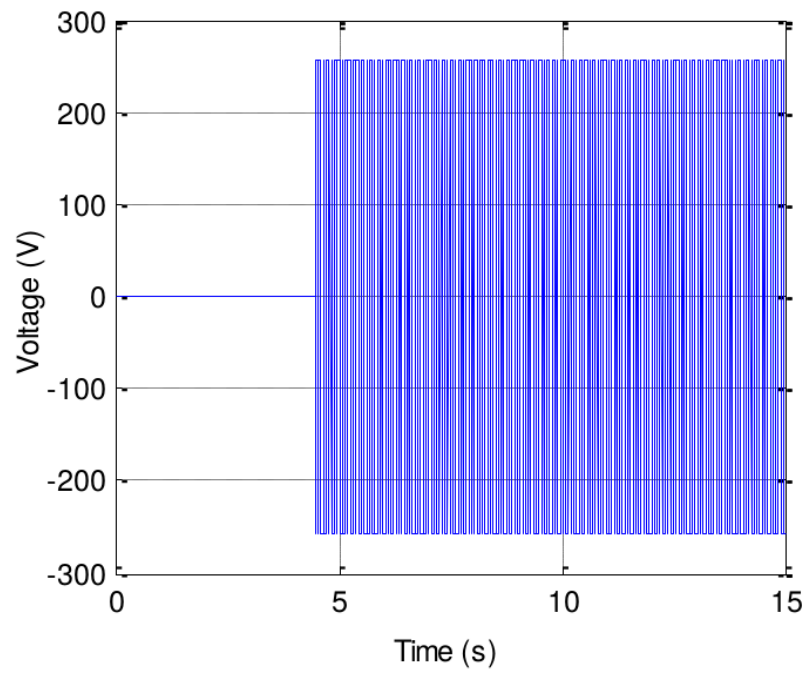

(c) Control voltage

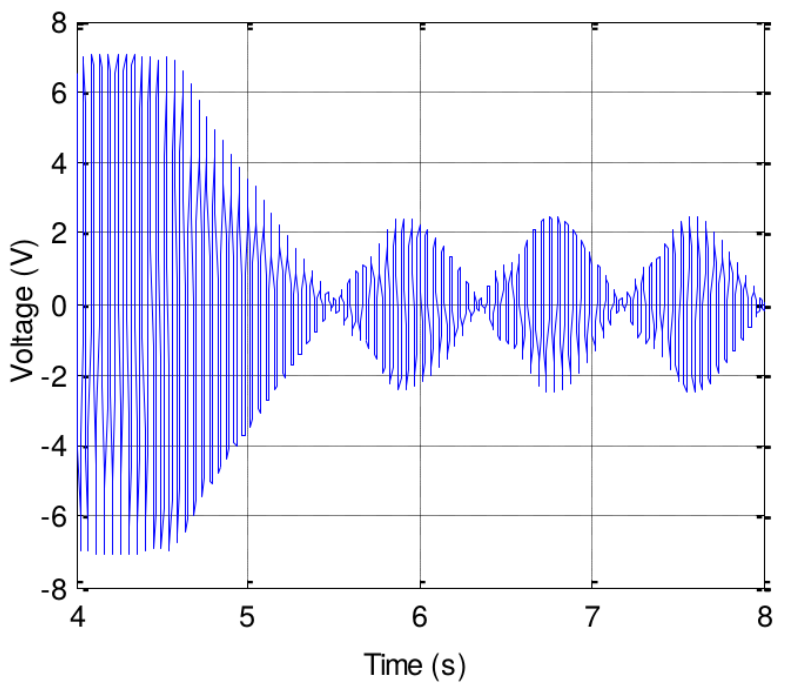

(b) Zoom in on the time axis of Fig. 12 (a)

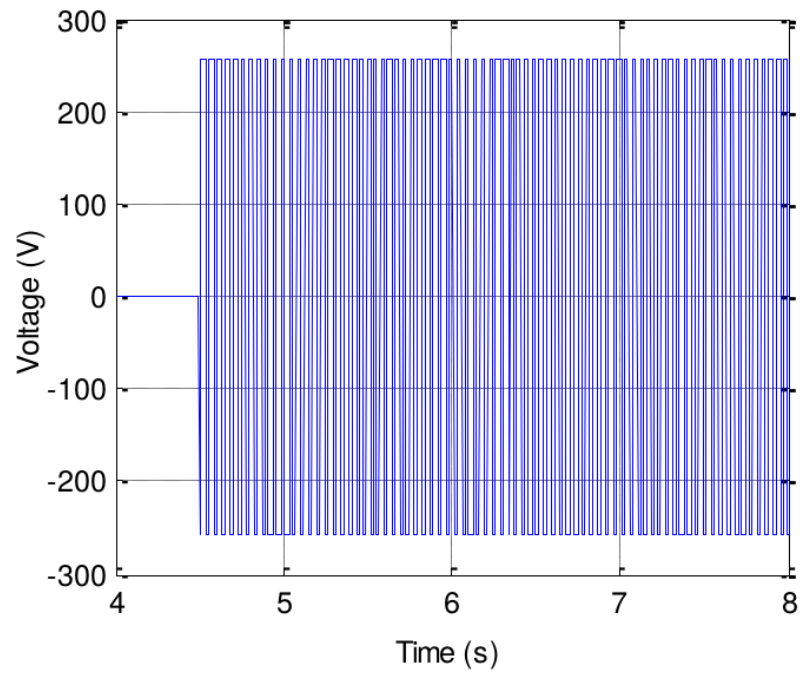

(d) Zoom in on the time axis of Fig. 12 (c)

Figure 12. Experimental result of switch variable structure control.

This is the difference between the acceleration sensor-based proportional-integral feedback control algorithm and the subsequent variable structure control algorithm. The experimental results demonstrate the effectiveness of the acceleration sensor-based proportional-integral feedback control algorithm controller. Furthermore, the experimental results are in good accordance with the simulation results to some extent.

When using the switch variable structure control algorithm to suppress the resonant vibration, the constants are chosen as $c_{1}=0.8, c_{2}=0.02$. Figure 12 (a) shows the time-domain resonant vibration response before and after control. Figure 12 (c) shows the control voltage applied on the PZT actuators. Figures 12 (b) and (d) are the enlarged view of Figs. 12 (a) and (c) from 4 seconds to 8 seconds, respectively. When the control action is not applied, the vibration amplitude is about $7 \mathrm{~V}$. After the active control is applied, the vibration is suppressed quickly. When the vibration is stable, the vibration amplitude increases and decreases periodically. The maximum vibration amplitude of the stable vibration response is about $2.5 \mathrm{~V}$. As depicted in Fig. 12 (c), when the active control is applied, the control voltage is saturated continuously at a high frequency.

When using the improved variable structure control algorithm, the parameters are selected as $c_{1}=0.6, c_{2}=0.04$, $\epsilon=2.5$, and $q=1.8$. Figure 13 (a) shows the time-domain resonant vibration response before and after control. Figure 13 (c) shows the control voltage applied on the PZT actuators. Figures 13 (b) and 13 (d) are the enlarged view of Figs. 13 (a) and (c) from 4 seconds to 8 seconds, respectively. After the active control is applied, the vibration is suppressed. When the vibration is stable, the vibration amplitude increases and decreases periodically, and the maximum vibration amplitude is $1.6 \mathrm{~V}$. As depicted in Fig. 13 (c), when the active control is applied, the control voltage is saturated continuously. This is why there is a building time where switch does not happen. The experimental results are in good accordance with the simulation results to some degree.

Remarks: The parameters of the designed controllers are provided by trial and error method in simulations and experiments. To guarantee the stability conditions of the controllers, control spill-over for higher-mode vibrations should be consid- 


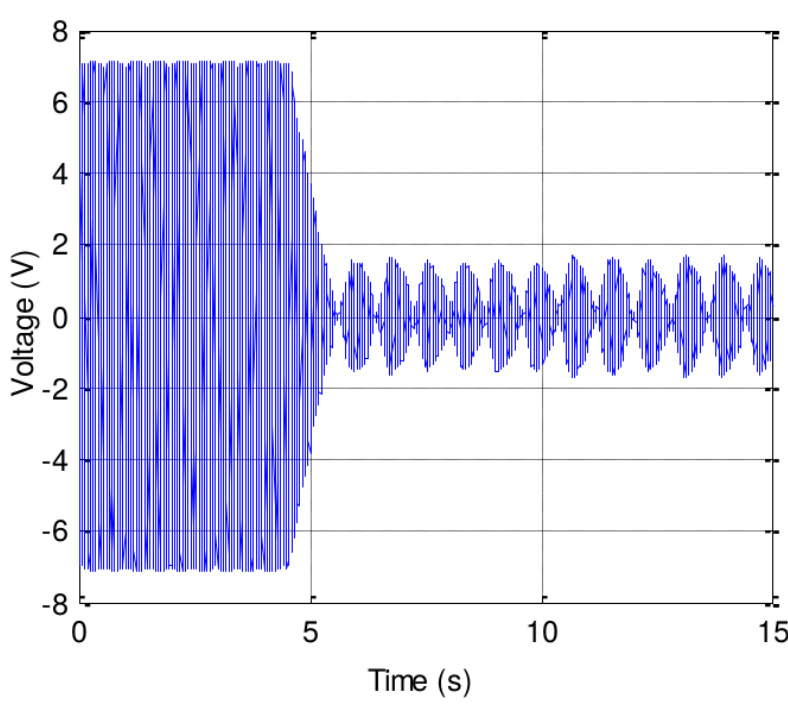

(a) Resonant vibration before and after control

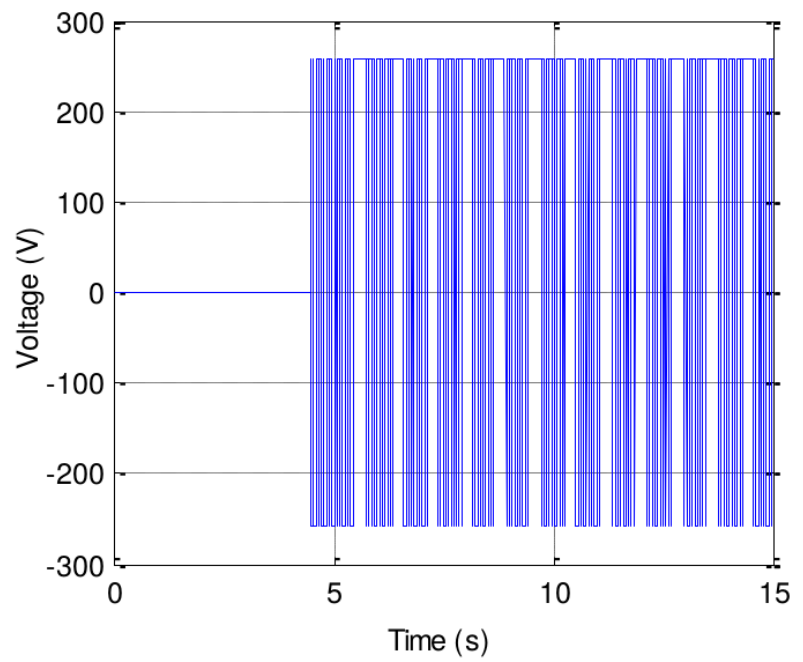

(c) Control voltage

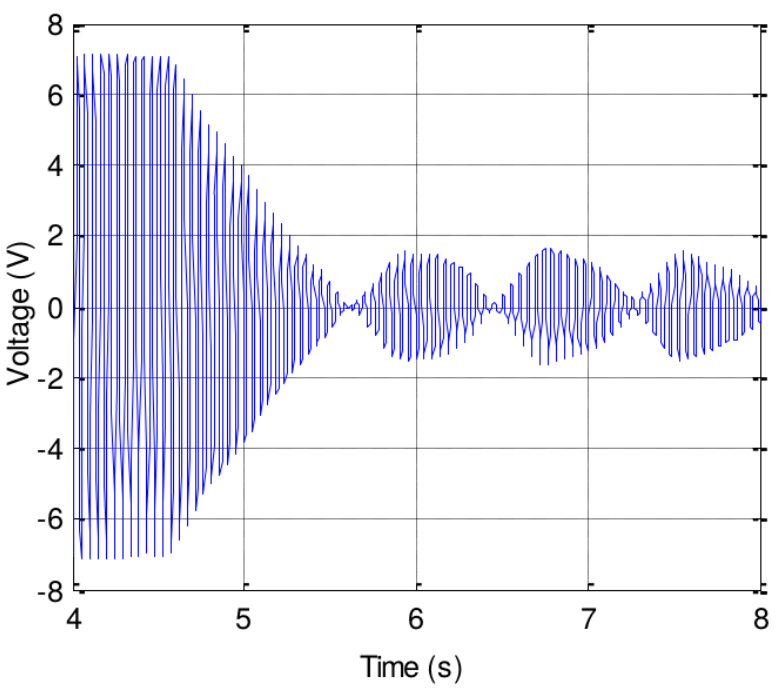

(b) Zoom in on the time axis of Fig. 13 (a)

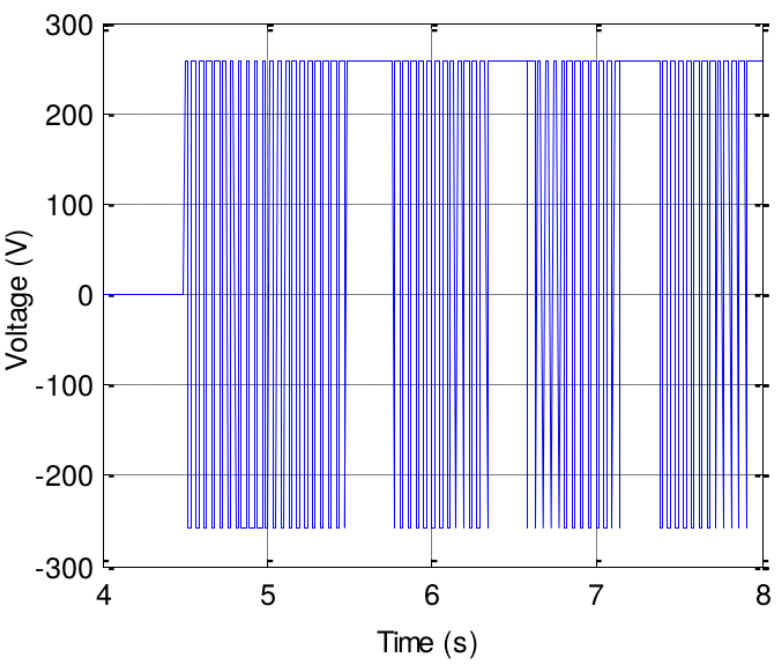

(d) Zoom in on the time axis of Fig. 13 (c)

Figure 13. Experimental result of improved variable structure control.

ered. Here, low-pass filters are applied to prevent spill-over.

From the experimental results, it can be concluded that the acceleration feedback-based control algorithm can suppress the resonant vibration effectively. In addition, the improved variable structure control algorithm shows a better performance in these proposed methods.

\section{CONCLUSIONS}

This paper presents the theoretical analysis and experimental results of the first resonant vibration suppression of a doubly-clamped flexible beam with bonded discrete PZT actuators and a mounted accelerometer. The dynamics model of the flexible beam system is obtained by using the finite element method. Acceleration sensor-based proportional-integral control method and sliding mode variable structure control algorithms are used to suppress the resonant vibration of the beam excited by piezoelectric actuators. Numerical simulations and experiments are conducted to compare the results of the different control methods. The resonant vibration of the doublyclamped beam is effectively suppressed by the proposed meth- ods. The experimental results confirm the effectiveness and robustness of the presented acceleration sensor-based control strategies. What's more, the improved variable structure control method shows better control performance in suppressing the resonant vibration.

\section{ACKNOWLEDGEMENTS}

This work was partially supported by the National Natural Science Foundation of China under Grant 51175181, partially supported by the Fundamental Research Funds for the Central Universities SCUT, and in part by State Key Laboratory of Robotics Foundation. The authors gratefully acknowledge these support agencies.

\section{REFERENCES}

1 Dwivedy, S. K. and Eberhard, P. Dynamic analysis of flexible manipulators, a literature review, Mech. Mach. Theory, 41 (7), 749-777, (2006). http://dx.doi.org/10.1016/j.mechmachtheory.2006.01.014 
2 Lin, J. and Liu, W. Z. Experimental evaluation of a piezoelectric vibration absorber using a simplified fuzzy controller in a cantilever beam, J. Sound Vib., 296 (3), 567-582, (2006). http://dx.doi.org/10.1016/j.jsv.2006.01.066

3 Chang, W., Gopinathan, S. V., Varadan, V. V., and Varadan, V. K. Design of robust vibration controller for a smart panel using finite element model, J. Vib. Acoust., 124 (2), 265276, (2002). http://dx.doi.org/10.1115/1.1448319

4 Hurlebaus, S. and Gaul, L. Smart structure dynamiequations (12) and (13)cs, Mech. Syst. Signal Pr., 20 (2), 255-281, (2006). http://dx.doi.org/10.1016/j.ymssp.2005.08.025

5 Wang, S., Quek, S., Ang, K. Vibration control of smart piezoelectric composite plates, Smart Mater. Struct., 10 (4), 637, (2001). http://dx.doi.org/10.1088/0964-1726/10/4/306

6 Sabatini, M., Gasbarri, P., Monti, R., and Palmerini, G. B. Vibration control of a flexible space manipulator during on orbit operations, Acta Astronaut., 73 (4), 109-121, (2012). http://dx.doi.org/10.1016/j.actaastro.2011.11.012

7 Vasques, C. H., da Conceição, S. M., de Abreu, G. L. C. M., et al. Identification and vibration control of a flexible structure, ABCM Symposium Series in Mechatronics, 4 (2), 157-165, (2012).

8 Parameswaran, A. P. and Gangadharan, K. Active vibration control of a smart cantilever beam at resonance: A comparison between conventional and real time control, Proc. Intelligent Systems Design and Applications (ISDA), Kochi, India, (2012), 235-239. http://dx.doi.org/10.1109/isda.2012.6416543

9 Mahmoodi, S. N. and Ahmadian, M. Modified acceleration feedback for active vibration control of aerospace structures, Smart Mater. Struct., 19 (6), 065015, (2010). http://dx.doi.org/10.1088/0964-1726/19/6/065015

10 Nima Mahmoodi, S., Craft, M. J., Southward, S. C., and Ahmadian, M. Active vibration control using optimized modified acceleration feedback with adaptive line enhancer for frequency tracking, J. Sound Vib., 330 (7), 1300-1311, (2011). http://dx.doi.org/10.1016/j.jsv.2010.10.013

11 Kwak, S. K., Washington, G., and Yedavalli R. K. Acceleration feedback-based active and passive vibration control of landing gear components, J. Aerospace Eng., 15 (1), 1-9, (2002). http://dx.doi.org/10.1061/(asce)08931321(2002)15:1(1)

12 Preumont, A. and Loix, N. Active damping of a stiff beamlike structure with acceleration feedback, Exp. Mech., 34 (1), 23-26, (1994). http://dx.doi.org/10.1007/bf02328438

13 Gatti, G., Brennan, M. J., and Gardonio, P. Active damping of a beam using a physically collocated accelerometer and piezoelectric patch actuator, J. Sound Vib., 303 (3), 798813, (2007). http://dx.doi.org/10.1016/j.jsv.2007.02.006
14 Shin, C., Hong C., and Jeong W. B. Active vibration control of beam structures using acceleration feedback control with piezoceramic actuators, J. Sound Vib., 331 (6), 1257-1269, (2012). http://dx.doi.org/10.1016/j.jsv.2011.11.004

15 Qiu, Z. C., Han, J. D., Zhang, X. M., et al. Active vibration control of a flexible beam using a noncollocated acceleration sensor and piezoelectric patch actuator, J. Sound Vib., 326 (3), 438-455, (2009). http://dx.doi.org/10.1016/j.jsv.2009.05.034

16 Qiu, Z. C., Wu, H. X., and Ye, C. D. Acceleration sensors based modal identification and active vibration control of flexible smart cantilever plate, Aerosp. Sci. Tech., 13 (6), 277-290, (2009). http://dx.doi.org/10.1016/j.ast.2009.05.003

17 Chatterjee, S. Vibration control by recursive time-delayed acceleration feedback, J. Sound Vib., 317 (1), 67-90, (2008). http://dx.doi.org/10.1016/j.jsv.2008.03.020

18 Ramu, I. and Mohanty, S. Study on free vibration analysis of rectangular plate structures using finite element method, Procedia Eng., 38, 2758-2766, (2012). http://dx.doi.org/10.1016/j.proeng.2012.06.323

19 Alkhatib, R. and Golnaraghi, M. Active structural vibration control: a review, Shock Vib. Digest, 35 (5), 367-383, (2003). http://dx.doi.org/10.1177/05831024030355002

20 Benjeddou, A. Advances in piezoelectric finite element modeling of adaptive structural elements: a survey, Comput. Struct., 2000, 76 (1), 347-363. http://dx.doi.org/10.1016/s0045-7949(99)00151-0

21 Yasin, M. Y., Ahmad N., and Alam, M. N. Finite element analysis of actively controlled smart plate with patched actuators and sensors, Lat. Am J. Solids Struct., 7 (3), 227-247, (2010). http://dx.doi.org/10.1590/s167978252010000300001

22 Lam, K., Peng, X., Liu G., and Reddy, J. N. A finite-element model for piezoelectric composite laminates, Smart Mater. Struct., 6 (5), 583-591, (1997). http://dx.doi.org/10.1088/0964-1726/6/5/009

23 Bandyopadhyay, B., Manjunath, T. C., and Umapathy, M. Modeling, control and implementation of smart structures: a FEM-state space approach, Springer-Verlag, Berlin, (2007).

24 Hung, J. Y., Gao W., and Hung J. C. Variable structure control: a survey, IEEE Transactions on Indust. Elect., 1993, 40 (1), 2-22. http://dx.doi.org/10.1109/41.184817

25 Fung, E. H. and Lee, C. K. Variable structure tracking control of a single-link flexible arm using time varying sliding surface, J. Robotic Syst., 1999, 16 (12), 715-726. http://dx.doi.org/10.1002/(sici)10974563(199912)16:12<715::aid-rob4>3.3.co;2-y 